\title{
Reformando la educación: lecciones de Estados Unidos, Finlandia, Corea del Sur, Brasil y Chile ${ }^{1}$
}

\author{
Transforming Education: Lessons from the \\ United States, Finland, South Korea, Brazil \\ and Chile
}

\section{Reformando a educação: lições de Estados Unidos, Finlândia, Coréia do Sul, Brasil e Chile}

\author{
Boris Salazar Trujillo \\ Docente e investigador de la Universidad del Valle, Cali, Colombia \\ boris.salazar@correounivalle.edu.co

\section{Luis Aurelio Ordóñez ${ }^{3}$} \\ Docente e investigador de la Universidad del Valle, Cali, Colombia \\ luis.ordonez@correounivalle.edu.co
}

1 Los autores agradecen la asistencia de Lina Marcela Franco y Edwin Caleño en el trabajo de investigación, y la financiación de la Rectoría de la Universidad del Valle.

2 Economista. Magíster en Economía.

3 Economista. Magíster en Historia. 


\title{
Resumen
}

Aunque no hay un método óptimo para evaluar las políticas o reformas educativas de largo plazo, creemos que es posible encontrar un conjunto de regularidades en la dinámica de los procesos de reforma, y sobre esa base evaluar su flexibilidad, energía transformadora, coherencia y capacidad para movilizar la mayor cantidad de recursos sociales, humanos, intelectuales, económicos y de cooperación. Encontramos que las reformas exitosas son producto de la acción y no del diseño humano, y que la flexibilidad, la participación y cohesión sociales, y la adopción de sistemas adaptativos complejos están en la base de las reformas exitosas y duraderas. Por el contrario, las reformas fallidas han elegido principios inflexibles y abstractos. Finlandia es un buen ejemplo de las primeras; Chile y Corea, de las fallidas. Estados Unidos es un caso aparte: ha logrado mantener su ventaja en la educación superior, a pesar de la debilidad de su educación básica.

Palabras clave: reforma educativa; educación superior; sistemas adaptativos complejos.

Clasificación JEL: I21, I23, I28.

\begin{abstract}
Although there is no a definitive method to appraise long-run educational reforms or policies, we claim that it is possible to find a set of regularities concerning the dynamics of educational reforms so as to assess their flexibility, transformational power, coherence and capacity to mobilize the highest amount of social, human, intellectual, economic and cooperative resources. We found that successful and deeply transformational reforms have been the product of human action and not of human design, and that flexibility, social cohesion, community involvement and the adoption of complex adaptive systems are the foundations for successful and enduring educational reform. Finland is a good example of the first type, Chile and Korea of failed reforms. and the United States is a special case, since it has managed to maintain its advantage in higher education, despite the weakness of their basic education.
\end{abstract}

Keywords: Educational reform, higher education, complex adaptive systems.

\section{Resumo}

Embora não exista um método ótimo para avaliar as políticas ou reformas educativas de longo prazo, acreditamos que é possível encontrar um conjunto de regularidades na dinâmica dos processos de reforma. Assim sendo, pode-se avaliar sua flexibilidade, sua energia transformadora, coerência e capacidade para mobilizar a maior quantidade de recursos sociais, humanos, intelectuais, econômicos e de cooperação. Encontramos que as reformas exitosas e constantes são produto da ação e não do desenho humano, e que a flexibilidade, a participação e coesão social, assim como a adoção de sistemas adaptativos complexos estão na base de estas reformas. Então, as reformas fracassadas têm elegido princípios inflexíveis e abstratos. Finlândia é um bom exemplo das primeiras, enquanto Coréia do Sul e Chile das segundas. Estados Unidos é um caso diferente: tem conseguido manter sua vantagem na educação superior, a pesar da debilidade da sua educação básica.

Palavras-chave: reforma educativa; educação superior; sistemas adaptativos complexos.

Este trabajo está bajo la licencia Creative Commons Attribution 3.0

¿Cómo citar este artículo? / How to quote this article?

Salazar, Boris y Luis Ordóñez. «Reformando la educación: lecciones de Estados Unidos, Finlandia,

Corea del Sur, Brasil y Chile». Sociedad y economía, No. 30 (enero - junio de 2016): 171-195. 


\section{Introducción}

Nadie ha inventado una fórmula definitiva para evaluar las reformas educativas. Ni antes ni después de su ejecución. No es posible predecir cuáles serán sus efectos futuros, como tampoco es posible adjudicar, con un grado mínimo de consenso, las causas que llevaron a su éxito o fracaso. Entre otras razones, porque el éxito arrollador de hoy podría convertirse en el fracaso aterrador de mañana, y los errores del pasado podrían devenir la esperanza del futuro en unas pocas décadas.

Pero establecer la imposibilidad de una fórmula definitiva no es equivalente a postular la inutilidad del estudio analítico de los grandes procesos de cambio en las políticas educativas, emprendidos en distintos Estados nacionales a lo largo de los dos últimos siglos. Nuestra hipótesis es que es posible encontrar algunos patrones básicos en la dinámica de los distintos procesos de reforma educativa. Si bien esos patrones no se repiten con exactitud de ley, sí es posible encontrar, en los distintos procesos estudiados, relaciones entre variables, contextos y políticas que dan origen a los mismos problemas y tensiones en distintos procesos.

Por eso, sugerimos estudiar un conjunto de problemas que reflejan los conflictos, las tensiones, las posibilidades y las restricciones básicas de toda reforma educativa. Frente a la ignorancia total, o al lienzo vacío sobre el que podrían escribirse los más bellos caracteres, preferimos estudiar un conjunto de interacciones que conduce a problemas y conflictos susceptibles de soluciones diversas.

Una vez detectados los grandes problemas, corresponde enfrentar la mayor dificultad de todas: generar las soluciones concretas que podrían poner una nueva política educativa sobre una trayectoria específica, entre las varias disponibles o imaginables. Es allí donde naciones y comunidades deben enfrentar la prueba más dura: la que separa las reformas fallidas, o inocuas, de las significativas o transformadoras.

¿Cuáles serían los criterios objetivos con los que podríamos juzgar una reforma como inocua o transformadora? No hay, otra vez, un conjunto de principios universalmente aceptados que permitan separar, con un margen reducido de error, unos procesos de otros. Es inevitable, entonces, elegir un conjunto de principios lo más universal posible —aunque siempre esté sujeto a disputa- para intentar la separación de los procesos transformadores de los inocuos, sin importar la ideología que los ilumine. Pero la elección de ese conjunto de principios supone, sin duda, realizar elecciones situadas en el dominio de lo ideológico.

¿Es universal, por ejemplo, elegir la igualdad como el principio básico de toda reforma educativa? ¿Igualdad de qué -de acceso a la mejor educación disponible, o a cualquier tipo de educación? ¿Igualdad para acceder a lo mejor de la cultura y de la ciencia? ¿O igualdad de oportunidades? La igualdad en abstracto podría ser aceptada por todos, siempre y cuando la discusión respecto a los mecanismos de adjudicación de la igualdad quedara por fuera del escenario.

Aquí aparece el problema de las relaciones entre principios y mecanismos que estará en el centro de la metodología que proponemos en este trabajo. Si el principio universal de igualdad estuviese conectado a un mecanismo de mercado en el que solo los más talentosos fueran seleccionados para acceder a la 
mejor educación posible y los demás fueran asignados a trayectorias educativas más limitadas, con menores recursos docentes y tecnológicos, menores exigencias y menor valoración de la autonomía y de la creatividad individuales, el resultado final sería una mayor desigualdad.

El punto central es que incluso los principios más universales, y con mayor tradición en la cultura igualitaria del iluminismo, al ser implementados mediante mecanismos que promueven la desigualdad, terminan generando, al ser aplicados al mundo real, exactamente lo contrario de lo que deberían haber inducido. La consecuencia de esta observación es simple pero decisiva para nuestro ejercicio: no es posible evaluar la pertinencia o la capacidad transformadora de una política educativa a partir de los puros principios aducidos en sus motivaciones. Es indispensable estudiar los efectos de las interacciones entre los principios postulados y los mecanismos elegidos para concretarlos. No hacerlo implicaría caer en una discusión estéril sobre principios básicos o, peor aún, sobre prejuicios ideológicos que dejaría fuera de foco los espacios en los que las verdaderas diferencias, problemas y tensiones están localizados.

Más aún: las trayectorias reales de las reformas educativas no siguen procesos determinísticos en los que las semillas iniciales (principios, inversiones, mecanismos) llevarían a los sistemas educativos por rutas conocidas y predecibles. Esa confianza ilimitada en la capacidad de predicción de modelos y mecanismos ha conducido a graves distorsiones de los procesos educativos, de la igualdad y la cohesión sociales. Sus efectos reales sugieren que muchas de las predicciones de los modelos más determinísticos han fallado significativamente.

En realidad, la interacción entre principios, mecanismos y eventos, propia de toda reforma, desencadena procesos complejos de adaptación que pueden producir resultados diversos de acuerdo con su capacidad de aprendizaje y de cambio. Durante su desarrollo, gobiernos, educadores, estudiantes, empresarios, intelectuales y padres de familia deben tomar decisiones sobre la marcha para corregir, o profundizar, la trayectoria tomada por la reforma en la que participan. Si esas decisiones tienen el momento apropiado para mantener el ritmo de cambio requerido por el proceso, tenderán a consolidarse en instituciones, normas, estructuras y actividades.

Aquí aparece la importancia de la flexibilidad en el desarrollo de las reformas educativas. Por ejemplo, los procesos menos ligados a ideales fijos de mercado o de control estatal han exhibido mayor flexibilidad, capacidad de cambio y poder de movilización de los recursos sociales y humanos requeridos para su desarrollo. A pesar de sus obvias diferencias, los procesos de cambio de Estados Unidos y Finlandia son buenos ejemplos de flexibilidad, iniciativa descentralizada y adaptación, logrados por vías distintas.

Por el contrario, los procesos de Chile y Corea del Sur muestran las dificultades asociadas con procesos inflexibles, confianza ciega en mecanismos económicos de dudosa implementación y fuerte sesgo ideológico. En general, los procesos con menor capacidad de aprendizaje y flexibilidad para tomar caminos distintos en los momentos decisivos terminan produciendo resultados negativos: movilizan menos recursos, profundizan la desigualdad y son menos sostenibles. 


\section{Diseño humano versus acción humana}

En algunos trabajos académicos ha comenzado a abrirse paso la hipótesis de que las grandes universidades del mundo son sistemas adaptativos complejos, que no siguen un plan definido en su desarrollo, sino que a través de procesos de ensayo y error encuentran soluciones emergentes para su avance.

Susanne Lohmann (2006), por ejemplo, ha defendido con brillantez la hipótesis de que las universidades públicas de investigación son sistemas adaptativos complejos y no, empresas económicas que deberían seguir las reglas de la administración corporativa. Lo que los críticos encuentran más ineficiente y ofensivo en la administración y el desarrollo de las grandes universidades, para Lohmann es lo que las hace un producto único de la evolución social y del aprendizaje humano, logrado en procesos que ocupan varios siglos y se remontan a la vieja universidad medieval.

El fundamento esencial del argumento de Lohmann es que la gran universidad de investigación no es producto del diseño humano, sino de la acción humana (Lohmann 2006, 2). No se trata de un simple cambio de sustantivos. Postular que las grandes universidades son producto del diseño humano supondría que sus formas actuales de gobierno, administración y desarrollo, incluidos su tamaño y carácter holístico, habrían surgido de las ideas y de la prodigiosa capacidad de predicción de las mentes privilegiadas que las crearon en los siglos XIX y XX. Pero es imposible adjudicar la autoría de las distintas dimensiones que conforman la universidad de investigación de hoy. No hay trayectorias que conduzcan a los textos, discursos, proyectos y leyes en los que estarían previstos lo que hoy constituye la estructura y la actividad de esas grandes instituciones.

Como lo plantea Lohmann, algunos de los atributos esenciales de la universidad de hoy tienen sus orígenes en diseños surgidos en la Europa medieval, entre ellos su autonomía en materia de gobierno, pensamiento y organización interna:
La idea de la universidad emergió, manifestándose en las normas de ubique docendi (el derecho a enseñar en cualquier institución una vez graduado en una de ellas), li- bre acceso, información abierta y libre examen. Estas normas -tan poderosas como lo son- derivan en últimas su poder de las estructuras institucionales de la univer- sidad: una norma de libre examen no vale mucho sin una estructura que proteja al que investiga de ser apresado, muerto o (peor aún) excomulgado (Lohmann 2006, 4, latín en el original).

La combinación de estas normas con la investigación básica, proveniente de la universidad alemana del siglo XIX, y con la mezcla de esta y de las grandes universidades holísticas de Estados Unidos, y luego con la financiación estatal a la investigación de punta realizada en las universidades, generaron la gran universidad de investigación que hoy conocemos. Nadie habría podido imaginar el resultado final de proceso tan largo y complejo.

Gustavo Rojas (2005) sugiere una visión optimista del cambio adaptativo de las instituciones universitarias en la era de la comunicación total en redes. Al aumentar en forma exponencial los vínculos entre las universidades y su entorno, y entre ellas mismas, la información tendería a disiparse hacia otras esferas 
sociales, distantes del equilibrio, produciendo nuevas estructuras y formas organizativas a través de la interacción no lineal y espontánea entre universidades y estructuras sociales:

\begin{abstract}
Un proceso causal mutuo de amplificación de las desviaciones, a través de bucles de retroalimentación positiva. Así considerado, este sistema es un sistema de acción que introduce y amplifica la innovación en las distintas regiones de su entorno. Su acción no es incoherente, como el foco de luz que irradia en todas las direcciones; antes bien se asemeja a la acción coherente del láser, pudiendo direccionar (sic) su actividad sobre regiones específicas del meta-ambiente, en las cuales amplifica la magnitud de las fluctuaciones, desencadenando, bajo ciertas condiciones, procesos de reacción en cadena (Rojas 2005, 364).
\end{abstract}

Más allá de los aspectos controversiales de la propuesta de Rojas (2005), solo queremos subrayar la importancia de la interacción creciente entre niveles educativos, estructuras sociales y regiones en un proceso de reforma educativa. Interacciones inéditas entre la más avanzada educación superior y la educación primaria y secundaria (incluso preescolar) -en la forma de cursos, aprendizaje por problemas, y participación de niños, jóvenes y maestros en proyectos científicos de punta- podrían acelerar el momento requerido para avanzar hacia una reforma educativa efectiva.

El plan brasilero (Lula da Silva, Haddad y Nicolelis 2008), que intenta combinar la ciencia más avanzada y la población más vulnerable en procesos educativos desplegados en todo el país, privilegiando las regiones más deprimidas, es un buen ejemplo de cómo la interacción entre tecnologías de punta, política social, coherencia educativa y poblaciones vulnerables puede generar trayectorias inéditas de reforma educativa y mayor cohesión social.

La distinción entre acción y diseño humanos es decisiva para comprender la tensión básica existente entre dos visiones opuestas de las reformas educativas. La dominante, a la que convergen partidarios de los mecanismos de mercado y del control estatal, postula que las reformas son el resultado del diseño humano. Supone que si las organizaciones, los funcionarios y los intelectuales encargados de imaginar y negociar las reformas encuentran el mejor diseño posible, su aplicación juiciosa será suficiente para alcanzar resultados óptimos en el futuro.

La visión alternativa sugiere que las reformas educativas son producto de la acción humana que aprende -a través del ensayo y el error, y de la combinación de iniciativas, normas y estructuras- a encontrar trayectorias hacia el cambio educativo, la igualdad y el conocimiento. Pero el hallazgo de esas trayectorias tampoco asegura nada. Incluso las mejores ideas, los procesos más flexibles, y las instituciones más dúctiles y efectivas son susceptibles de cambio y están siempre abiertas a la crítica y, a veces, al asalto violento de ideologías fundamentalistas.

Diane Ravitch plantea con elocuencia la difícil relación entre fines y medios en el estudio de las reformas educativas, y el altísimo costo de asumir posiciones flexibles:

Crónicas periodísticas afirmaban que yo había hecho un giro en U, que me había echado para atrás en todo lo que creía. No era cierto. Había cambiado de opinión acerca de los medios, no de los fines. Mi filosofía educativa básica seguía siendo la 
misma: siempre había deseado que todos los niños pudieran tener la misma educación de alta calidad que deseaba para mis hijos y nietos. (...) Eventualmente, me di cuenta de que el acoplamiento entre mercados libres y responsabilidad basada en exámenes no resolvería nuestros males educativos y que, de hecho, amenazaba con hacerlos peores (Ravitch 2010, 243) .

Tres reglas provisionales de método emergen aquí. La primera es que estudiar los principios sin tener en cuenta los mecanismos elegidos es un ejercicio inútil. En materia de reformas educativas, el estudio de las tensiones ideológicas puras, basadas en el análisis crítico de principios fundamentales, no permite detectar las diferencias auténticas ni entender las trayectorias de sus efectos.

La segunda es que las interacciones entre principios y mecanismos -en contextos políticos, sociales y culturales diversos- conducen a situaciones específicas, susceptibles de tratamiento analítico mediante la detección de patrones comunes que conducen a uno u otro tipo de trayectoria dinámica. Allí está la clave para entender las trayectorias cambiantes de las políticas educativas. Sistemas construidos a partir de principios y conjuntos de incentivos similares pueden adoptar trayectorias distintas de acuerdo con las decisiones de política tomadas en las encrucijadas producidas por las interacciones entre principios, mecanismos y eventos externos al sistema mismo.

La tercera es que las reformas más efectivas, y con más efectos transformadores, emergen en momentos en los que naciones y sociedades enfrentan encrucijadas decisivas. No es extraño, pues, que los giros más fuertes en política educativa hayan ocurrido después de guerras civiles y mundiales, del ascenso de dictaduras, de grandes movimientos sociales, del final sangriento de grandes experimentos de cambio político y social, en grandes crisis económicas y en momentos de optimismo o de cambio.

El proceso de desarrollo de las universidades de investigación en Estados Unidos permite ver los alcances de las interacciones entre principios, mecanismos y eventos desde una perspectiva de larga duración. Aunque parezca extraño, las guerras desempeñaron un papel fundamental en las trayectorias tomadas por el sistema de investigación más fuerte del mundo. Tanto la guerra civil estadounidense como las dos guerras mundiales y la de Vietnam fueron decisivas en los giros de política, que cambiaron para siempre la trayectoria del sistema de universidades de investigación de Estados Unidos. En el centro de esos giros está el papel cambiante - tanto en magnitud como en orientación- del Estado, o del gobierno federal, en su diseño y desarrollo.

El nacimiento de lo que sería la gran universidad de investigación estadounidense fue el conjunto de incentivos establecidos por el Congreso en plena guerra civil. Los objetivos del Congreso eran expandir el sistema nacional de educación e innovación y mejorar la calidad de la educación superior y la investigación. Según Cole $(2010,28)$, las leyes Morrill de 1862 y Hatch de 1887 fueron las más importantes de un amplio conjunto de leyes que puso las bases para el desarrollo del sistema de educación superior de Estados Unidos.

4 Todas las traducciones de citas originales en inglés fueron realizadas por Boris Salazar. 
La primera proveyó las bases legales para la construcción de lo que sería uno de los atributos fundamentales del sistema estadounidense -el campus universitario- a través de la entrega gratuita de terrenos federales para la creación de universidades y colegios públicos. Aunque en un principio las instituciones creadas estaban ligadas al desarrollo de tecnología y la investigación relacionadas con la agricultura, con el tiempo se transformaron en universidades estatales completas con miles de estudiantes cada una. La creación de dos instituciones fundamentales para la puesta en marcha de un sistema nacional de educación superior y de investigación -la Fundación Nacional para la Ciencia (NSF), en 1950, y la expansión de los institutos nacionales de la salud (NIH), en 1947- cerraron el entramado institucional de la reforma.

Los principios básicos que fundamentaban la política adoptada fueron más o menos universales: universalidad educativa, coexistencia entre universidades estatales y privadas, búsqueda de la más alta calidad, énfasis en los efectos prácticos de la investigación y la innovación. Aunque esos principios, con variaciones, pueden ser encontrados en otros procesos nacionales, tres factores básicos distinguen el proceso estadounidense de los demás.

El primero es la interacción particular entre fondos públicos y las iniciativas privadas y sociales. Mientras el Estado federal proveía los terrenos y los fondos para financiar la investigación y la innovación, distintos ciudadanos y organizaciones privadas y comunitarias se unieron para crear universidades de investigación de alta calidad. La fortaleza y la viabilidad de tal sistema tuvieron una garantía fundamental en el carácter público tanto de las universidades estatales como de las privadas, en el sentido decisivo de que sus fondos provenían, y provienen, de la sociedad en su conjunto (impuestos o donaciones), y no están animadas por fines de lucro.

El segundo es que la competencia entre las universidades no estuvo centrada en la disminución de costos, la fijación de un precio único o la maximización de ganancias, como lo suponen las versiones vulgares de la adopción de mecanismos de mercado, sino en la búsqueda implacable de la más alta calidad. Fue un proceso natural de competencia basado en la búsqueda de objetivos propios de los procesos de educación, investigación y aplicación del conocimiento al desarrollo industrial, y no en la observación de criterios económicos abstractos 5 .

El tercero, y más estadounidense de todos, es el impacto de la acción de líderes educativos que combinaban, de forma inusual, grandes capacidades intelectuales con visiones audaces del futuro y habilidades gerenciales poco comunes, ilustrado muy bien por Vannevar Bush ${ }^{6}$, antiguo decano de Ingenierías de MIT,

5 Resulta irónico que las recomendaciones de política educativa, favorecidas hoy por el Banco Mundial y otras instituciones, bajo el dominio de la política exterior de Estados Unidos, estén centradas en criterios abstractos de eficiencia y competencia económicas, y no en los criterios educativos que guiaron la exitosa política de educación superior de ese país. Contrario a lo que cree el sentido común, la burocracia internacional no sigue la experiencia de Estados Unidos.

6 Jonathan Cole (2010) retrata en detalle el personaje que fue Bush y su influencia prodigiosa sobre el desarrollo de las grandes universidades de Estados Unidos. Este pasaje condensa muy bien el significado de Bush en ese logro: "Vannevar Bush fue un gigante. Al igual que Benjamin Franklin, combinó la agudeza científica y tecnológica con una visión de cómo podían ser usadas al servicio de propósitos nacionales" (Cole 2010, 87). 
quien como presidente del Consejo Nacional para la Investigación en Defensa (NDRC) tuvo a su cargo el diseño de las instituciones de investigación con las que Estados Unidos enfrentaría, después de la Segunda Guerra Mundial, la competencia científico-política con la antigua Unión Soviética (Atkinson y Blanpied 2008; Cole 2010).

\section{El papel de lo nacional}

En todos los procesos de reforma educativa o de transformación radical de las políticas educativas que hemos estudiado es evidente el papel de lo nacional en las soluciones ideadas para los problemas educativos de cada país. Por nacional no entendemos la identidad de cada pueblo, sino la forma en que cada sociedad, al pensarse como nación, afronta el problema de cómo educar a sus ciudadanos. Solo las sociedades que han intentado resolver su problema educativo como parte de un propósito nacional de transformación han tenido algún éxito en el logro de sus objetivos.

Lo nacional no es una declaración de principios; es una fuerza que anima el conjunto del proyecto, fija sus alcances, y pone en tensión los recursos económicos, mentales, físicos y éticos necesarios para establecer la línea de cambio elegida. De hecho, es el resultado de la convergencia de trayectorias históricas, imaginarios nacionales y perspectivas culturales sobre cierta coyuntura. Lo nacional está en la forma específica en que una sociedad detecta, piensa, moviliza y resuelve, colectivamente, cómo educar a sus habitantes.

Esto no quiere decir que el papel de lo nacional sea idéntico en todos los procesos estudiados. Al contrario, la forma en que cada uno aborda el problema educativo desde lo nacional permite diferenciarlos en forma más o menos clara. El sistema de educación superior de Estados Unidos —el más grande y potente del mundo- no es comparable, ni en su trayectoria ni en sus logros ni en su combinación de incentivos económicos y políticas públicas, a ninguno otro en el mundo. Es un sistema único, tanto por el momento en que surgió como por las fuerzas que lo conformaron, las alianzas tempranas con la industria y la innovación que lo hicieron posible, el tamaño geográfico y poblacional del país, y su capacidad de adaptación a eventos históricos impredecibles.

Como sistema universitario de educación, el estadounidense surgió después del alemán y del inglés, y trató, en un principio, de imitarlos en lo fundamental. Pero las bases de su desarrollo posterior no estuvieron en la copia de los modelos originales, sino en la interacción novedosa entre políticas públicas, alianzas entre desarrollo económico y educación superior, comunidades y una autonomía extrema para las instituciones universitarias. Mientras el Estado cumplía la tarea básica de garantizar los terrenos y los recursos para la formación de universidades y colegios estatales, los hombres de negocios y los líderes educativos generaban las ideas y arriesgaban los capitales que habrían de conformar después de la Segunda Guerra Mundial el más formidable sistema de educación superior conocido en la historia, y las instituciones universitarias creaban su propio futuro en un contexto de libertad y autonomía difícil de encontrar en las recomendaciones de hoy.

La narrativa que Cole (2010) hace del proceso de emergencia de las grandes universidades de investigación muestra el papel del Estado federal y del Congreso en 
la promulgación de políticas básicas, y el enorme papel creativo de los líderes educativos y empresariales que forjaron esas grandes universidades a partir de ideas muy básicas y sin contar, en realidad, con un modelo a seguir. Más aún, muestra cuán importante fue la interacción puntual entre presidentes con una idea muy clara de lo que debería ser el futuro de la nación, y líderes educativos y empresariales con la capacidad de tomar decisiones muy rápidas y poner en marcha sistemas que, en su diseño, parecían ir en contra de sus creencias conservadoras. El ejemplo más claro es la interacción entre Franklin Delano Roosevelt y Vannevar Bush, de la que surgió la financiación estatal de la investigación básica y aplicada y la elección de las universidades más avanzadas y complejas como el agente que llevaría a cabo esa transformación.

Por su inicio temprano, la audacia con la que convirtió en oportunidades lo que parecían amenazas para su seguridad nacional y económica, y la liberación temprana de rendimientos crecientes, Estados Unidos explotó al máximo las potencialidades disponibles, dentro y fuera del país, en materia de talento humano, recursos físicos, coordinación, conocimiento y oportunidades.

Desde el punto de vista de la explotación espontánea de las capacidades de coordinación, invención, innovación y adaptación existentes en una sociedad, y en el mundo, el sistema de universidades de Estados Unidos es uno de los mejores ejemplos de la superioridad de los procesos espontáneos de uso de la información y de los recursos con respecto a alternativas centralizadas. Pero tiene un gravísimo problema: no es replicable. Poner a Estados Unidos como el objetivo a emular o seguir en el diseño de sistemas de educación superior es una ilusión costosa. $\mathrm{Ni}$ siquiera contando con la riqueza extrema y la escasa población de Arabia Saudita o de algunos de los pequeños reinos petroleros del Golfo Pérsico, un país podría construir un sistema de educación superior como el de Estados Unidos.

No es un asunto de puros recursos económicos. No hay ninguna cantidad de dinero que pueda comprar el flujo de estudiantes, profesores y científicos talentosos de todo el mundo que todos los años se matriculan en las universidades de Estados Unidos, y deciden permanecer allí. Dado el tamaño, los recursos, las conexiones y el valor agregado de la comunidad científica asociada a las universidades, laboratorios y firmas estadounidenses, la oferta salarial para que cualquier científico decidiera regresar a su patria debería cubrir, al menos, las ventajas asociadas a pertenecer a la red estadounidense de investigación e innovación. Mientras el número de conexiones, proyectos y científicos integrados a redes o sistemas alternativos no alcance cierto umbral crítico, será imposible que los científicos extranjeros regresen a sus países de origen.

El punto es que el conjunto de los sistemas universitarios del mundo conforma un sistema complejo ${ }^{7}$, dotado de rendimientos crecientes, que sigue una ley de potencia, en el que el triunfador se lleva casi todo, dejando solo las migajas para los demás competidores. Por eso, la probabilidad de que otro sistema nacional

7 Considerar todos los sistemas nacionales de educación como un sistema en su conjunto es una noción discutible. Es obvio que entre ellos no existen las interrelaciones y los vínculos para convertirlos en un sistema en el sentido estricto. Pero también es claro que los sistemas más desarrollados toman recursos (sobre todo humanos) de los menos desarrollados y que estos aprovechan, hasta donde pueden, los avances de los primeros. En todo caso, las interrelaciones existentes sí permiten hablar de un orden basado en las relaciones entre los sistemas nacionales. 
de educación e innovación alcance al de Estados Unidos, o pueda amenazarlo en forma efectiva, es cercana a cero. Y solo podría aumentar si algún choque desastroso del lado de la oferta (una quiebra colectiva de las universidades de investigación) o de la demanda (caída vertiginosa de la matrícula de estudiantes extranjeros talentosos y de la contratación de profesores y científicos extranjeros de talla mundial) ocurriera. En ese sentido, la estrategia de imitar al mejor no paga.

\section{Trayectorias, interacciones y encrucijadas}

Las trayectorias seguidas por las reformas o políticas educativas de largo plazo son el resultado de la interacción compleja entre niveles de inversión privada y estatal, movilización de los recursos sociales y privados, coherencia o integralidad del sistema educativo, inversión en capital docente, mecanismos de incentivos (económicos y no económicos), eventos históricos y contextos culturales y políticos.

Distintas combinaciones de niveles de esfuerzo en cada una de esas dimensiones generarán trayectorias también distintas. Un sistema con un alto nivel de inversión, pero incapaz de movilizar la cantidad suficiente de recursos intelectuales, económicos, éticos, políticos y sociales avanzará muy rápido en un comienzo, pero luego comenzará a perder fuerza hasta devenir insostenible. ¿Cómo es posible que la pura asignación de recursos económicos no garantice la máxima movilización de todos los recursos requeridos? Varias razones, o conjuntos de ellas, permiten explicar esa situación.

Una razón inmediata es la incapacidad para formar una coalición suficientemente grande de padres de familia, estudiantes, docentes, administradores educativos, hacedores de política, empresarios y comunidad en general comprometida con la reforma. Una reforma diseñada desde arriba y ejecutada sin la participación de las comunidades y de sus organizaciones habrá perdido el más importante de los recursos y deberá dedicar esfuerzos extras a la detección, análisis y corrección del problema. Si lo hace muy tarde, es probable que el hallazgo del problema ocurra en forma traumática, y llegue al mundo real como endeudamiento gravoso de individuos y hogares, mala calidad del sistema, desigualdad creciente y conflictos paralizantes.

El ejemplo de Finlandia es iluminador. Con su sistema político multipartidista, estaba mucho mejor preparada para la creación de consensos y coaliciones incluyentes que países donde el enfrentamiento entre dos grandes partidos o coaliciones o el fundamentalismo religioso hace, casi imposible la conformación de coaliciones nacionales (Antikainen y Luukkainen 2007). Israel —otro país líder en innovación y desarrollo- está hoy viviendo el desmoronamiento de su sistema de educación básica debido a las presiones de las sectas religiosas que dominan una parte creciente del electorado.

También es posible que la reforma exclusiva de un nivel educativo conduzca a la pérdida del esfuerzo con el que podrían contribuir los agentes que trabajan en otros niveles del sistema educativo. Por ejemplo, una reforma centrada en la educación superior, dejaría por fuera la contribución de las comunidades vinculadas a los niveles básicos del sistema educativo. En todos los casos, el proceso 
de reforma sufriría una filtración permanente de recursos, y sus efectos globales sobre el sistema educativo y sobre el bienestar y la igualdad sociales serían mucho menores.

Después de casi treinta años de predominio absoluto de la ideología del mercado en las instituciones internacionales y en las burocracias nacionales que decidían qué tipo de reforma seguir, resultaba casi natural pensar que la única forma de movilizar un máximo de recursos económicos y sociales era a través de mecanismos de mercado, incluyendo el uso de los fines de lucro. Los ideólogos de esta corriente pensaban que la pura movilización de capital privado y de los recursos de endeudamiento de los hogares era suficiente para comprometer un máximo de recursos y cambiar la educación. Lo que habría podido ser una hipótesis se convirtió en una ideología dominante, sin ningún tipo de control efectivo, ni desde el conocimiento ni desde la sociedad.

Tanto los defensores a ultranza de esta ideología como sus críticos más radicales cometen el error de creer que sus probables bondades así como sus notorias debilidades dependen del funcionamiento esencial del mercado. No es así. El problema esencial es que la educación no es un bien de consumo, transable en mercados competitivos donde agentes racionales comprarían y venderían "educación" hasta alcanzar el máximo excedente posible, producto de extraer la máxima disponibilidad a pagar de los compradores, y de encontrar los vendedores capaces de producir al costo más bajo posible.

Si el mercado funcionara tan bien como lo imaginan críticos y seguidores, el resultado sería desastroso para la educación y para la sociedad. Los individuos con mayor capacidad para pagar por el bien de consumo educación pagarían la mejor educación que sus bolsillos pudieran imaginar, y los demás tendrían que aceptar una educación a la altura de sus reducidos ingresos, o no tener ninguna. El resultado sería ineficiente, inequitativo y ruinoso. Ninguna sociedad ha tomado esta vía para educar a sus niños y jóvenes y para promover el bienestar social y la innovación. Los que optaron por trayectorias cercanas al ideal del mercado han sufrido las consecuencias destructoras sobre la educación, el aprendizaje, y la solidaridad y cohesión sociales. Chile es el caso emblemático, en Latinoamérica y en el mundo, de la aplicación optimista de este tipo de política (Klein 2007; Brunner y Uribe 2007).

De hecho, el mejor sistema de educación superior del mundo —el de Estados Unidos- se desarrolló bajo una política que iba, y va, en contravía de lo que aconsejaría el modelo de mercado de libro de texto de la burocracia internacional. Como lo han mostrado con suficiencia Gordon Winston y otros (Winston, Carbone y Lewis 1998; Winston 1996; 1997; 2001), la mejor educación es subsidiada por el Estado federal y por el público, el tamaño del subsidio es una función de su calidad y las universidades que la ofrecen gastan todos los recursos que reciben de la sociedad, sin tener en cuenta el principio de eficiencia. Como los clientes hacen parte de sus insumos, las mejores universidades compiten por atraer a los estudiantes más talentosos del mundo, al igual que a los profesores y científicos más productivos y prestigiosos.

El malestar creciente en la sociedad coreana, y los conflictos alrededor del endeudamiento social y la desigualdad en Chile, Gran Bretaña, Estados Unidos y otros países indican que combinar los incentivos de mercado, la movilización 
de capital privado y el endeudamiento de los hogares no es equivalente a la máxima movilización de los recursos sociales existentes. De hecho, la cantidad de recursos sociales movilizados es inferior al logrado a través de otras vías, y el nivel de riesgo lanzado sobre hogares e individuos, y sobre el sistema en conjunto, es mucho mayor que el asociado con otras alternativas. Incentivar la participación de todos en el esfuerzo educativo a través del endeudamiento solo podría funcionar, si hubiera un perfecto mecanismo de discriminación que únicamente les prestara a aquellos con mayor probabilidad de terminar sus estudios y lograr un empleo o devenir empresarios exitosos.

Pero la adopción de este mecanismo implicaría excluir del uso de los fondos estatales y privados a aquellos sin talento sobresaliente (o por descubrir), nacidos en hogares de menores ingresos, y provenientes de colegios y escuelas de menor calidad o con menores recursos. Más aún: para que el sistema funcionara desde el punto de vista financiero requeriría de la adopción de seguros contra la falla en el pago de las deudas por parte de los estudiantes y de los hogares comprometidos en préstamos educativos. Esos seguros, y la intermediación financiera privada, concentrarían los excedentes asociados al sistema en los bancos, compañías de seguros y fondos de inversión, logrando la fórmula perfecta para lograr la desigualdad más profunda y la destrucción de la solidaridad y la cohesión necesarias para conformar una sociedad viable y justa. En últimas, el arreglo social termina siendo inferior al que se quería mejorar, induciendo mayor desigualdad y empeorando a los más débiles.

\section{Sostenibilidad}

Una propiedad deseable de las trayectorias recorridas por las reformas educativas es que sean sostenibles en el tiempo. Como este no es el lugar propicio para discutir el concepto de sostenibilidad, el uso que hacemos aquí está circunscrito a los procesos de reforma educativa, y no pretende entrar en la complejidad de la discusión científica y política sobre el tema. Seguimos la idea más simple de sostenibilidad: "satisfacer las necesidades del presente, sin comprometer la capacidad de las generaciones futuras para hacerlo" (WCED 1987, 16).

Aunque simple y potente, su aplicación a la factibilidad de las reformas educativas exige algunos ajustes. El problema central de las reformas educativas no es conservar los recursos físicos y humanos de forma que las generaciones futuras tengan la posibilidad de un futuro al menos tan deseable como el nuestro. Es, más bien, conservar en el tiempo la capacidad de transformación imbuida en su despegue. Este concepto es, por supuesto, menos aprehensible desde el punto de vista cuantitativo. ¿Cómo medir el potencial de transformación de una reforma educativa? ¿Cómo medir su capacidad para conservar su impulso transformador? No hay, en principio, variables reales o índices construidos que permitan medir cantidad tan elusiva. Pero no es imposible aproximarla desde un punto de vista cualitativo.

Lo intentaremos analizando, primero, el papel de la relación entre el presente y el futuro en la sostenibilidad de las reformas educativas. El concepto de sostenibilidad clásico tiene la virtud de captar en forma precisa el problema de la solidaridad entre las generaciones presentes y las futuras y cómo el tipo de 
reforma puesta en marcha hoy determina la suerte, felicidad, supervivencia económica y el aprendizaje de las generaciones futuras. Una reforma sesgada hacia el endeudamiento real de individuos y hogares supone que la libertad de elegir de las generaciones futuras será mucho menor que la de las actuales, y que los estudiantes de hoy, y sus hijos, tendrán posibilidades mucho más restringidas que las disfrutadas por las generaciones presentes o por los encargados de diseñar la reforma en el pasado.

En términos más intuitivos, toda reforma educativa es una apuesta con respecto al futuro de una nación. Puede ser nacional si compromete a la coalición más grande posible en su diseño y ejecución, y si logra movilizar la mayor cantidad de recursos económicos, intelectuales y sociales en su realización (Salhberg 2012).

No se trata de construir mayorías. En condiciones de despotismo, las mayorías están siempre del lado de las reformas y podrían contribuir, con su silencio, a su puesta en marcha. Las grandes coaliciones unificadas alrededor de propósitos nacionales deberían ser el producto de grandes procesos de conversación, discusión, búsqueda y consolidación que solo son posibles en momentos de ampliación de la democracia. De hecho, la movilización de recursos potenciales no es el resultado de la simple aceptación pasiva de las líneas generales de una reforma, sino el descubrimiento de las potencialidades ocultas, y sin explotar, de una nación. Lo muestran las políticas exitosas, aunque opuestas, desplegadas en Estados Unidos y Finlandia.

Las diferencias son también importantes para la sostenibilidad de los procesos. El sistema estadounidense ha basado su superioridad en su poder para atraer a los más talentosos estudiantes y profesores del mundo, concentrando altísimas inversiones estatales y privadas en las universidades de investigación de elite, mientras deja las universidades públicas de investigación y los niveles de educación básicos a merced de las modas políticas de turno, disminuyendo sus recursos y promoviendo reformas que han ido minando la universalidad y el carácter público de la educación básica, y ha puesto contra la pared a las grandes universidades públicas de educación.

Las filtraciones inducidas en el sistema ya han comenzado a hacerse evidentes. Hay un déficit creciente de profesionales en sectores ligados al conocimiento y a las tecnologías más avanzadas (Goldin y Katz 2008; 2009), una caída en la formación de los estudiantes que emprenden estudios universitarios, mayores desigualdades entre universidades y entre colegios de secundaria y de primaria, endeudamiento creciente - tendiendo a lo catastrófico- de estudiantes y familias, y una pérdida de la coherencia del sistema en su conjunto, detectable en la puesta en marcha de reformas contradictorias, ligadas al ascenso de ideologías fundamentalistas y contrarias al libre examen y a la libertad de pensamiento.

La trayectoria de la reforma finlandesa ha sufrido menos filtraciones, y no ha dejado de movilizar una cantidad considerable de recursos, explotar los rendimientos crecientes del sistema adaptativo generado y descubrir nuevas posibilidades pedagógicas en el arreglo descentralizado elegido. Al contrario de lo sugerido por la ortodoxia, los sistemas financiados por el Estado no tienen que sufrir el destino inevitable de la centralización. En Finlandia la iniciativa local, la responsabilidad de cada profesor, equipo docente y escuela en el aprendizaje 
de sus estudiantes, y la confianza mutua han logrado mantener en marcha un sistema basado en la financiación estatal y en la iniciativa descentralizada de los agentes activos del sistema educativo.

Es importante subrayar que el sistema finlandés no nació descentralizado. Su deslizamiento hacia la descentralización fue producto del aprendizaje realizado en la década de 1970 y de los cambios inducidos en la de 1980. Antikainen y Luikanen describen así la mutación ocurrida en los años de 1980:

\begin{abstract}
Después del establecimiento del actual sistema finlandés de escuela integral en los años setenta y al principio de los ochenta, la administración de las escuelas integrales, y del sistema educativo en su conjunto, era muy centralizado. La centralización era considerada como el medio para realizar la reforma integral de la escuela, a pesar de la oposición de los partidos de derecha y de la asociación de profesores de secundaria. Al final de los años ochenta un cambio significativo ocurrió en la medida en que un paradigma de planificación incremental comenzó a ganar terreno y el sistema fue descentralizado. Las escuelas se volvieron más responsables de su propia administración y debieron construir su propio currículo según los lineamientos fijados por la Comisión Nacional de Educación (o Ministerio de Educación) (Antikainen y Luukkainen 2007, 2).
\end{abstract}

Para entender el éxito del paso de un sistema centralizado a uno descentralizado hay que tener en cuenta dos factores. El primero es la inmensa inversión en capital docente realizado por Finlandia, que garantizó un salto en el desempeño de los docentes, un cambio en su status social, y una confianza creciente en sus ideas e iniciativas. El segundo es la existencia en la sociedad finlandesa de grandes reservas de responsabilidad individual y de valoración del aprendizaje.

Sin embargo, la sostenibilidad del enfoque finlandés está hoy amenazada. No en su coherencia ni en la universalidad de su educación básica. En ese ámbito es claro que Finlandia ha elegido mantener una educación básica igual para todos y de la mejor calidad posible. Los resultados obtenidos por los jóvenes finlandeses en las pruebas PISA confirman la fuerza de un sistema en el que hay una sola prueba nacional y en el que la formación de los estudiantes no pasa por la presión de las pruebas y por la competencia. Los riesgos están en su sistema de educación superior. En un contexto global en el que la posición de las universidades depende de la magnitud y de la eficiencia de sus vínculos con la industria, en sectores en los que el conocimiento y las inversiones en investigación y desarrollo determinan cada vez más los resultados de la competencia, Finlandia ha tenido que tomar decisiones que hagan más competitivas a sus universidades, atraigan más capital extranjero, y consoliden alianzas y riesgos compartidos con capitales pertenecientes a las industrias de punta. Un antiguo ministro de Educación de Finlandia describía la nueva estrategia del gobierno así:

La financiación de la educación será desarrollada de forma que incentive el desempeño, con el fin de que cierta fracción de los fondos públicos sean asignados sobre la base del desempeño. Evaluar el desempeño ha desencadenado naturalmente un agitado debate alrededor de la dificultad de encontrar indicadores no controversiales y claramente justos. Hasta el momento, el gran éxito en esta área ha sido logrado en el sector universitario, aunque aún solo un mero 3\% del presupuesto ha sido asignado sobre la base del desempeño (Ollie-Peka 1997, 561). 
Desde una perspectiva no gubernamental, Antikainen y Luukkainen resumen los cambios y las amenazas fundamentales que hoy enfrenta el sistema finlandés:

Primero, el envejecimiento de la población y la migración han llevado al cierre de escuelas, en especial en las áreas rurales, pero también en las ciudades. Segundo, el aprendizaje en el trabajo y la cualificación basada en competencias han devenido más populares, y han fortalecido el poder de las asociaciones gremiales y corporativas y del llamado sistema tripartito (Estado, asociaciones y sindicatos de empleados). La migración y el cambio en la educación vocacional están, por supuesto, ligadas a la globalización, pero no en forma directa al neoliberalismo. Las estructuras básicas del modelo nórdico de educación integral están allí todavía, pero en una forma modificada y amenazada por procesos de reestructuración (Antikainen y Luukkainen 2007, 12).

\section{Trayectorias nacionales}

Si consideramos el conjunto de los sistemas de educación superior del mundo, es inevitable observar la posición dominante de Estados Unidos, en términos de inversión y calidad. Su trayectoria después de la mitad del siglo XIX y, sobre todo, después de la Segunda Guerra Mundial ha dominado a las de los demás países. Podría ser superada en inversión, pero es casi imposible que sea superada en calidad ${ }^{8}$. A menos, por supuesto, que el conjunto del sistema mundial cambiara y fueran modificados, también, los criterios con los que se evalúa la calidad. Aun si los científicos y graduados que hoy trabajan en Estados Unidos regresaran a la China y la India, y una fracción creciente de científicos y estudiantes decidieran estudiar y trabajar en esos países, y no en Estados Unidos, el panorama básico de hoy no cambiará en el futuro.

Las características nacionales 9 de Estados Unidos, Chile, Finlandia, Brasil y Corea del Sur ${ }^{10}$, los eventos que precedieron a los grandes giros en sus políticas educativas y las elecciones políticas realizadas por sus sociedades permiten explicar sus trayectorias. Estas son el resultado de la interacción entre niveles elegidos de inversión en educación y en capital docente, grados distintos de descentralización de los procesos educativos, coherencia entre los distintos niveles del sistema educativo y compromiso temprano con la innovación como parte de la cultura nacional.

No todos han seguido la misma trayectoria. El nivel de inversión, por ejemplo, depende de los incentivos elegidos, del compromiso del Estado con la reforma educativa, del uso de incentivos de mercado en su implementación y de

8 Medida en la forma impuesta por el mismo modelo estadounidense de grandes universidades de investigación: calidad de profesores y estudiantes, productividad de los profesores, flujo de innovaciones, impacto de la investigación realizada. El impacto de la docencia no cuenta en este tipo de medición.

9 Los países seleccionados fueron elegidos por haber realizado reformas radicales en sus sistemas educativos, el éxito de sus reformas, la influencia que han tenido sobre el resto del mundo y el carácter divergente de sus trayectorias.

10 En la muestra original también estaba incluido Singapur, pero no pudimos conseguir información de la misma calidad que la encontrada para los otros países. Por eso, el conjunto de países estudiados en detalle quedó reducido a Estados Unidos, Chile, Brasil, Corea del Sur y Finlandia. 
los mecanismos de financiación. Las decisiones tomadas en cada uno de esos campos conforman un sistema que tiene efectos más allá del ámbito educativo, afectan la igualdad y el bienestar de todos, y conducen a cada experiencia nacional por trayectorias distintas.

Los datos muestran que entre 1997 y 2007 la relación entre financiación pública y privada cayó de 0,52 a 0,36 (tabla 1). Aquí aparece la esencia del modelo estadounidense de desarrollo de la educación superior, fundamentado en una inversión privada que se convierte en pública en la medida en que está conformada por donaciones y filantropía, que llegan desde la sociedad civil, y en una educación de muy alta calidad y muy excluyente por su altísimo costo y su estrategia de aceptar solo a los más talentosos del mundo.

Tabla 1. Proporción pública/privada de la inversión en educación

\begin{tabular}{|c|c|c|c|c|}
\hline \multicolumn{5}{|c|}{ Proporción pública/privada de la inversión global en educación } \\
\hline Años & Chile & Finlandia & Corea & Estados Unidos \\
\hline 1997 & - & - & & 0,52 \\
1998 & 1,25 & 6,44 & 1,27 & 0,53 \\
1999 & 1,21 & - & 1,30 & 0,54 \\
2000 & 1,16 & - & - & 0,34 \\
2001 & 1,28 & 28,00 & 1,10 & 0,50 \\
2002 & 1,21 & 28,07 & - & 0,50 \\
2003 & 1,06 & 28,38 & 1,22 & 0,50 \\
2004 & 1,06 & 27,82 & 1,24 & 0,42 \\
2005 & 1,13 & 26,33 & 1,22 & 0,43 \\
2006 & 1,29 & 23,29 & 0,89 & 0,48 \\
2007 & 1,45 & 23,51 & 0,84 & 0,36 \\
\hline
\end{tabular}

Fuente: OECD 2011.

Es una concepción de lo público fundamentada en la sociedad civil, en las decisiones libres de los individuos, en la existencia de lazos comunitarios y afectivos entre las universidades y sus egresados y en un papel creciente del Estado, después de la Segunda Guerra Mundial, en la financiación de las áreas de investigación de punta. De hecho, todos los caminos exitosos en educación en el mundo han sido públicos, así tengan fuertes mecanismos de mercado en su implementación. Lo han sido el coreano, el estadounidense, el finlandés y hasta el brasileño y el chileno, cada uno a su manera. No lo son, por supuesto, desde el punto de vista del valor de la educación que ofrece.

Finlandia está situado en el otro extremo del espectro manteniendo, a lo largo de los 8 años para los que hay datos, proporciones que van desde 28 , 01 en 2001 hasta 23,5 en 2007. Ha habido una disminución, pero la proporción entre lo estatal y lo privado sigue siendo la más alta de todas.

En Chile la proporción entre el gasto público y el privado en educación ha crecido lentamente, como reflejo de su política de financiar, con fondos estatales, a operadores privados y de subsidiar a través de la demanda. Corea, en cambio, ha reducido en forma sistemática la participación estatal en la financiación 
de la educación, invirtiendo la proporción hasta llegar a poner solo 84 centavos de dólar por cada dólar invertido por el sector privado.

En general, la efectividad de la inversión educativa depende de la coherencia del sistema educativo en su conjunto. Si la inversión crece sin que la coherencia del sistema lo haga al mismo tiempo, la efectividad de la inversión caerá: cada estudiante que arribe a la educación superior dotado de una formación básica incompleta representará una filtración para el sistema en su conjunto. Una fracción de cada peso invertido en él o ella se perderá irremediablemente en ese conjunto de procesos que llamamos deserción, y que no son más que el efecto de las desigualdades sociales, reforzadas por una educación primaria y secundaria deficientes, y por arreglos sociales que inducen la desigualdad.

\section{La apuesta coreana}

Para entender la complejidad del problema que estamos planteando, consideren la estrategia de cambio educativo de Corea del Sur. A comienzos de los años de 1960, Corea era un país rural, tan atrasado o más que Colombia. Sin embargo, a partir de ese momento sus historias divergen en forma radical. Después del fin de la traumática ocupación japonesa, Corea apostó a la recuperación de su maltrecha identidad nacional a través de la educación. La reconstrucción, desde la base, de su sistema educativo fue el centro de su estrategia de recuperación de la identidad nacional. El honor jugó un papel central en su implementación. Educar a los coreanos se convirtió no solo en tarea de educadores, administradores y burócratas estatales, sino en el deber superior de todas las familias coreanas. El honor familiar estaba en juego en dos dimensiones: en su papel en la reconstrucción de la identidad coreana y en su obligación de garantizar la mejor educación posible para sus hijos. Esa obligación no fue entregada a colegios y a educadores. Cada padre y madre de familia debía adoptarla como su deber básico con la patria y con sus valores ancestrales.

Siguiendo el buen ejemplo de Estados Unidos, y adaptándolo a las condiciones coreanas, los reformadores tomaron una versión transformada de la competencia estadounidense y la unieron con el desarrollo de grandes conglomerados industriales. Lo han estado haciendo a través de dos agentes de cambio. Las familias no han dejado de invertir una fracción creciente de sus recursos para que sus hijos sean admitidos en las mejores instituciones educativas y logren una posición en alguno de los conglomerados industriales. Esa inversión va más allá de la pura educación formal y cubre la contratación de tutores en casa y en lugares especializados, el seguimiento continuo de su progreso y el sacrificio de las horas de entretenimiento de las familias.

La estrategia concertada entre el Estado y la gran industria innovadora condujeron a la construcción de un sistema educativo que absorbe a los mejores y más talentosos en los conglomerados responsables de la innovación, mientras las universidades garantizan el flujo de aplicaciones requerido para mantener en marcha el sistema de industrialización dirigido a la exportación.

La búsqueda de una mejor posición dentro del sistema mundial de educación caracterizó los años de 1990, junto al arribo del síndrome de la reforma. 
Como lo sugiere Cheng (2009), Corea no escapó al miedo a la pérdida de competitividad, por lo que inició una serie de reformas orientadas a garantizar la calidad, mejorar el control y evaluación de las escuelas y consolidar la visión de un sistema educativo concentrado en la producción de servicios educativos para una fuerza laboral latente.

El nuevo milenio trajo consigo nuevos desafíos. El impacto de la revolución de la información es evidente en la tercera etapa de la reforma coreana, en la que el papel de la educación como la herramienta con la que se prepara a los mejores del futuro alcanza un lugar preponderante. Los mejores ya no compiten dentro de las fronteras nacionales, sino que deben hacerlo con sus pares en los mercados internacionales. Para enfrentar el problema de la competitividad a escala global, Corea introdujo el séptimo currículo, cuyo objetivo era hacer comparables las capacidades y competencias de los graduados coreanos con las de los mejores del mundo.

Es evidente que el sistema coreano intensifica, hasta niveles que bordean la obsesión, el sistema estadounidense de competencia y búsqueda implacable de la calidad. El uso de pruebas estandarizadas desde la educación primaria garantiza la elección de los más talentosos, disciplinados y obedientes mediante el uso de un instrumento en apariencia objetivo. Lo mismo ocurre con colegios y universidades. Una vez alcanzada alguna ventaja en las pruebas, los mayores recursos estatales y el prestigio creciente tienden a retroalimentarse a través de la elección de los mejores estudiantes a todos los niveles. Mejores estudiantes garantizan mejores resultados para sus colegios y universidades. Las mejores notas garantizan mayores recursos y prestigio y estos garantizan, otra vez, la absorción de los mejores estudiantes de las nuevas cohortes. El sistema se reproduce a sí mismo en forma predecible y eficiente.

Pero no es igual al estadounidense en dos factores fundamentales. El sistema de educación superior de Estados Unidos no es eficiente. Dada su posición dominante en el mundo, el flujo de recursos hacia las mejores universidades de investigación no depende de su eficiencia, sino de su prestigio. Es posible que esas grandes universidades dilapiden recursos en investigaciones inútiles, en ciencias excéntricas, en disciplinas olvidadas o en innovaciones destinadas al fracaso, pero ningún otro sistema educativo del mundo tiene el prestigio suficiente para que esos fondos y ese talento fluyan hacia ellos en la misma proporción.

El punto es que el sistema de Estados Unidos puede darse el lujo de no ser eficiente, sin que su posición dominante corra peligro. Por dos razones básicas. La primera es que su sistema no requiere de la coherencia entre los distintos niveles educativos nacionales y puede darse el lujo de tener resultados promedio mediocres en las pruebas Pisa. Esa debilidad la resuelve importando los estudiantes y científicos más talentosos del mundo. La otra es que las universidades pueden gastar todos los fondos que reciben del Estado, de donantes privados y ciudadanos de todo el mundo, mientras esos individuos e instituciones estén dispuestos a seguir haciéndolo. Todo depende de cuánto quieran seguir invirtiendo burócratas y capitalistas en las grandes universidades de investigación. Mientras lo continúen haciendo al ritmo de hoy, el 
"ineficiente" sistema norteamericano seguirá siendo el mejor del mundo." Lo que lleva, de paso, a una conclusión provisional: la alta calidad en la educación superior es contradictoria con la eficiencia económica.

Tratar de seguir la estrategia del líder, sin serlo, tiene consecuencias graves. La primera es que no hay ningún nivel de inversión que permita alcanzar al primero en términos de calidad y de innovación. La segunda es que si bien la estrategia puede funcionar en corto y en el mediano plazo, en el largo plazo, al alcanzar el máximo de su esfuerzo, comienza un declive expresado en menores rendimientos a la educación superior, cansancio, frustración y resistencia creciente de los jóvenes a participar en la carrera propuesta por su sistema educativo nacional. Los datos de cobertura en educación superior son elocuentes: un impresionante $63 \%$ de los coreanos entre 25 y 34 años tienen un grado universitario -la tasa más alta de los países de la OECD- y 71\% de los coreanos en edad de hacerlo entra a la universidad (Cheng 2009, 78).

Pero los efectos sobre el empleo y la sostenibilidad de conjunto de la estrategia no son positivos. El incremento en la proporción de graduados universitarios ha conducido a mayores tasas de desempleo para los recién graduados, y a la pérdida de capital, ingresos y propiedad para los hogares cuya inversión no ha sido premiada con el éxito de sus hijos. El desbalance entre la inversión realizada y los ingresos recibidos conduce a pérdida colectiva de recursos, caída de las expectativas y depresión creciente.

Hay un trade-off, entonces, entre los buenos resultados inmediatos y de mediano plazo de las reformas basadas en mecanismos de mercado y en la selección sistemática de los mejores, de un lado, y los resultados inferiores, en el corto y mediano plazo, pero superiores en el largo plazo, de reformas basadas en la búsqueda de la igualdad y la descentralización de los esfuerzos, del otro. Pensamos en el ejemplo de Finlandia, que siguió una estrategia integral para todo el sistema, confiando en la cooperación, la búsqueda descentralizada de soluciones y la iniciativa local, de la mano con fuertes inversiones en la formación de capital docente en todos los niveles.

\section{Finlandia: un camino excéntrico hacia lo mejor}

La trayectoria adoptada por Finlandia rompe con todos los patrones establecidos, cuestiona la convergencia inevitable hacia las reformas educativas basadas en el mercado y las pruebas estandarizadas, y sugiere que hay otros caminos exitosos hacia el cielo prometido del cambio social a través de la educación (Sahlberg 2011; 2012). Lo original no está en sus logros, sino en la trayectoria elegida para alcanzarlos. En lugar de seguir el camino de las reformas basadas en la estandarización, la competencia, la responsabilidad de los administradores académicos y la financiación privada, Finlandia eligió un camino inédito con resultados sorprendentes. Sus elecciones, los momentos en que decidió hacerlo, y los altos grados de cooperación, igualdad, flexibilidad y descentralización

11 Las grandes universidades de Estados Unidos tienen unos de los portafolios de inversión más rentables del mundo. Por su gigantesco tamaño pueden contratar a los expertos más prestigiosos y elegir las estrategias de inversión más audaces (Piketty 2014, 447-452). 
desplegados hacen pensar en un proceso recursivo en el que las transformaciones educativas han impulsado, y han sido impulsadas, por profundas transformaciones sociales, teniendo como fundamento la activación de atributos normativos y éticos presentes en la cultura finlandesa.

A diferencia del tipo de reforma dominante en el mundo, la estrategia finlandesa no buscó la energía social necesaria para emprender una reforma de esa magnitud en la aplicación abstracta de incentivos y mecanismos de mercado, sino en las capacidades para acordar, cooperar, crear y confiar existentes en potencia en la sociedad. Mientras que las reformas basadas en el mercado solo confían en la efectividad de la búsqueda de ganancias privadas y en la competencia ilimitada, la reforma finlandesa buscó sus recursos básicos en la capacidad de los finlandeses para convertir la cooperación, la flexibilidad y la confianza mutua en atributos de un proceso permanente de cambio.

Quizás el aislamiento en el que vivía Finlandia, la presencia de una fuerte ética del trabajo y del esfuerzo, y la propensión a cooperar en empresas colectivas la salvaron de seguir el camino elegido por otras naciones o de haber adoptado, unos años más tarde, las estrategias del movimiento para la reforma global de la educación que hoy domina en el mundo (Sahlberg 2007).

$\mathrm{Al}$ camino tomado por la experiencia finlandesa confluyen varios factores: su aislamiento relativo, la desaparición de la Unión Soviética y la fuerte recesión económica que la acompañó, una sólida ética del trabajo, una cultura que privilegia lo colectivo sobre lo individual, una sociedad más compacta que la mayoría de las sociedades del mundo, altas dosis de confianza mutua y una antigua valoración de la lectura como medio para alcanzar otros fines (Oppenheimer 2010; Sahlberg 2007).

Es una combinación exitosa de eventos espontáneos, desastres económicos convertidos en oportunidades y una muy fuerte propensión a cooperar. A pesar de las diferencias culturales, geopolíticas y sociales, la experiencia israelí es comparable con la finlandesa en varios aspectos: países pequeños, aislados del mundo exterior, con escasos recursos naturales, fuerte propensión a cooperar y a la igualdad, y capacidad para convertir las crisis en oportunidades. Ambas naciones han logrado convertir sus debilidades en fortalezas, desarrollando fuertes culturas de la innovación y situándose entre las primeras del mundo en inversión en investigación y desarrollo, y en producción de patentes por número de habitantes.

¿Cuál es clave para la exitosa desviación de Finlandia? Como lo plantea Sahlberg $(2007,150)$, es difícil señalar las reformas o innovaciones puntuales que habrían puesto en marcha las fuerzas que han elevado el nivel y la calidad del aprendizaje en Finlandia. Al estar ligados por múltiples lazos, es difícil discernir cuáles serían las reformas puramente educativas que explicarían los extraordinarios resultados de los estudiantes finlandeses. Más aún: la clave del éxito no está en ciertos factores individuales, o de conjunto, sino en el proceso mismo. Sahlberg, citando a Aho et al. (2006), sugiere una hipótesis que apunta en esa dirección: "Más que sobre la introducción de reformas e innovaciones secuenciales, la política educativa finlandesa ha sido edificada sobre un liderazgo sostenible, fundamentado en valores comunes y una visión compartida" (Traducción propia) (2006, 149-150).

Las nociones de liderazgo sostenible y de valores y visiones comunes parecen demasiado gaseosas al ser enfrentadas con las piedras angulares de la reforma 
global: estandarización del desempeño de estudiantes y profesores sobre la base de los resultados obtenidos en un número creciente de pruebas; concentración absoluta en matemáticas y lenguaje (lectura y escritura) y responsabilidad total del desempeño de escuelas y estudiantes sobre administradores y profesores, siguiendo una lógica estricta de la supervivencia de los mejores.

Es obvio que las reformas basadas en esos principios básicos tienen la ventaja insuperable de producir sus propias estadísticas y mejorar su propio desempeño una vez establecidos los criterios de competencia. Es ideal para producir efectos inmediatos, promover el prestigio de sus líderes, sintonizar a la opinión pública y generar una mística ciudadana alrededor de la educación. Pero, ¿es sostenible? Después de los brillantes fuegos artificiales de los primeros años, ¿qué es lo que se conserva? A juzgar por los resultados de varios países, entre ellos Estados Unidos (Ravitch 2010), no mucho: el impulso inicial parece que no se sostiene en el largo plazo.

¿Qué ha hecho factible la trayectoria finlandesa? Más que en factores específicos, la clave está en los procesos desatados. Sí, no hay duda de que la alta inversión en capital docente, la elevación del estatus social y económico de los profesores, la existencia de un currículo nacional flexible, la asignación a los profesores de la responsabilidad de los procesos de aprendizaje y de evaluación han contribuido puntualmente a la factibilidad de la trayectoria finlandesa.

Sin embargo, lo que ha permitido la contribución de cada uno de sus factores al desarrollo exitoso de la estrategia ha sido la puesta en marcha de procesos sociales basados en la confianza, la igualdad, la flexibilidad y la responsabilidad inteligente. En el centro de esos procesos ha estado el aprendizaje. Los logros en cobertura, en los resultados de las pruebas, y en el desempeño de escuelas, colegios y universidades han llegado luego como un efecto inevitable pero no buscado del proceso puesto en marcha. Lo que importa no son tanto los fines, sino los procesos desatados y sostenidos en el tiempo. El alcance de los fines llega por sí solo, en forma espontánea, como lo condensa Sahlberg en una frase afortunada: "Como nación de gentes modestas, Finlandia nunca se propuso, en realidad, ser la mejor del mundo en educación" $(2011,41)$.

La inversión de lugar entre fines y procesos ha sido fundamental. Lo que ha movido al conjunto de estudiantes, profesores, administradores, padres de familia y ciudadanos en general no son las metas fijadas por el Estado, o por los líderes empresariales y educativos, sino el deseo de aprender dentro de condiciones apropiadas para el aprendizaje. En lugar de perseguir el alcance de metas óptimas de desempeño, los finlandeses desencadenaron un proceso endógeno de transformación de las opiniones, creencias y paradigmas que dominaban las prácticas pedagógicas.

Más que tratar de imponer sus puntos de vista o sus metas, los reformadores hicieron posible un espacio en el que profesores, administradores y estudiantes podían buscar sus propias vías hacia el aprendizaje a través de procesos de ensayo y error. De allí surgieron mejores prácticas y métodos exitosos que luego fueron compartidos a través de redes de apoyo y retroalimentación que, con el tiempo, generaron un sistema nacional de aprendizaje y evaluación espontáneos. 
Transversal a todo el proceso, ha estado la activación de un conjunto de propensiones y de valores éticos y culturales que han permitido la movilización de recursos sociales que resultarían invisibles para estrategias basadas en puros incentivos privados. Es obvio que los valores y propensiones éticas y culturales que han coadyuvado al proceso finlandés no son replicables en otros lugares del mundo. Sin embargo, constituyen un horizonte fundamental para la construcción de reformas educativas en cualquier lugar del mundo.

\section{Conclusiones}

Las reformas educativas transformadoras no son obra del diseño humano, sino de la acción colectiva de múltiples actores en procesos adaptativos complejos. La clave de su éxito está en las interacciones entre principios, incentivos y eventos, y en la flexibilidad con la que comunidades, maestros, administradores educativos, estudiantes, padres de familia, intelectuales, funcionarios estatales y empresarios toman, en forma descentralizada, las decisiones que permiten mantener el momento transformador de la reforma puesta en marcha.

Los efectos de largo plazo de las reformas estudiadas muestran la superioridad de los logros obtenidos por reformas que siguieron procesos adaptativos complejos, en los que coaliciones de educadores, estudiantes, padres de familia, intelectuales, ciudadanos y comunidades aprendieron, sobre la marcha, a corregir el rumbo, convertir los problemas en oportunidades, y seguir un propósito nacional definido. Por el contrario, reformas basadas en principios inflexibles, ya sea de mercado o contrarios a él, han producido arreglos sociales inferiores en el largo plazo. El caso de Finlandia es un buen ejemplo de las primeras; Corea y Chile de las segundas.

Las reformas educativas deben ser sostenibles. Es decir, ser capaces de mantener su impulso transformador en el complejo proceso de su despliegue. Es un concepto dinámico de conservación. No se trata de conservar los mismos recursos y conocimientos, sino de sostener el momento de cambio del proceso iniciado. Esa sostenibilidad depende de las condiciones iniciales inducidas en el sistema, y de la fuerza de la iniciativa descentralizada puesta en marcha.

Una reforma transformadora requiere de una interacción positiva entre igualdad y coherencia en la educación. Igualdad no es equivalente a mayor cobertura. Supone que todos los niños tienen derecho a la mejor educación básica posible, no a cualquier educación. En el sistema finlandés son nueve años de escolarización básica a la que todos deben tener acceso en forma gratuita. La coherencia entre todos los niveles del sistema educativo requiere de la igualdad, y la igualdad solo es posible garantizando la coherencia del conjunto del sistema desde los niveles más básicos. El ideal de la excelencia solo es factible si está presente desde el punto de entrada al sistema educativo. Otra vez el énfasis está en los procesos y no en el diseño. Es el impulso generado en los niveles básicos del sistema el que garantizará el momento necesario para que los niveles superiores crezcan en forma armónica. Más y mejores estudiantes no aceptarán una educación mediocre. 


\section{Referencias bibliográficas}

Antikainen, Ari y Anne Luukkainen. Twenty-Five Years of Educational Reform Initiatives in Finland, 2007. http://cc.joensuu.fi/ anti/publ/uudet/twenty_five_ years.pdf (último acceso: 15 de noviembre de 2014).

Atkinson, Richard y William Blampied. «Research Universities: Core of the US Science and Technology System». Technology in Society, $\mathrm{n}^{\circ}$ 30, 2008: 30-48.

Barnett, Ronald. Los límites de la competencia, el conocimiento, la educación superior y la sociedad. Barcelona: Gedisa, 2001.

Bok, Derek. Universidades a la venta. Comercialización de la educación superior. Valencia: Universitat de Valencia, Guada, 2010.

Brunner, José y Daniel Uribe. Mercados universitarios: el nuevo escenario de la educación superior. Santiago de Chile: Universidad Diego Portales, 2007.

Cheng, Yin. «Teacher Management and Educational Reforms: Paradigm Shifts». Prospects, $\mathrm{n}^{\circ}$ 39, 2009: 69-89.

Cole, Jonathan. The Great American University. Nueva York: Public Affairs, 2010.

Goldin, Claudia y Lawrence Katz. The Race Between Education and Technology. Cambridge, MA: Belknap Press, 2008.

Goldin, Claudia y Lawrence Katz. «The Future of Inequality: The Other Reason Education Matters so Much». Milken Institute Review, $\mathrm{n}^{\circ} \mathrm{ll}$ (Third Quarter), 2009: 26-33.

Haddad, Fernando. O Plano de desenvolvimento da educação: razões princípios e programas. Brasilia: Ministerio da Educaçao, 2007.

Klein, Naomi. The Shock Doctrine. The Rise of Disaster Capitalism. Nueva York: Picador, 2007.

Lohmann, Susanne. 2006. The Public Research University as a Complex Adaptive System. Ponencia presentada en "Proceedings of the European Conference on Complex Systems". Oxford, University of Oxford, 25-29 de septiembre.

Lula da Silva, Luiz, Fernando Haddad y Miguel Nicolelis. «Brazil's Option for Science Education». Scientific American, Vol. 298, n 2, 2008: 25.

Nussbaum, Martha. Sin fines de lucro. Por qué la democracia necesita de las humanidades. Bogotá: Katz, 2011.

OECD. Education at a Glance 2011: OECD Indicators, OECD Publishing, 2011. http://dx.doi.org/10.1787/eag-2011-en

Ollie-Pekka, Heinonen. «Finland: Restructuring Higher Education». Prospects, $\mathrm{n}^{\circ}$ 27, 1997: 559-569.

Oppenheimer, Andrés. ¡Basta de historias! La obsesión latinoamericana con el pasado y las 12 claves del futuro. Bogotá: Debate, 2010.

Piketty, Thomas. Capital in the Twenty-First Century. Cambridge, MA: Harvard University Press, 2014.

Ravitch, Diane. The Death and Life of the Great American School System: How Testing and Choice are Undermining Education. Nueva York: Basic Books, 2010.

Rojas, Gustavo. Modelos universitarios. Los rumbos alternativos de la universidad y la innovación. México D.F.: FCE, 2005.

Sahlberg, Pasi. «Education Policies for Raising Students Learning: the Finnish Approach». Journal of Education Policy, Vol. 22, $\mathrm{n}^{\circ}$ 2, 2007: 147-171.

Sahlberg, Pasi. Finnish Lessons. New York, NY: Teachers College Press, 2011. 
Sahlberg, Pasi. «A Model Lesson: Finland Shows Us What Equal Opportunity Means». American Educator, Spring 2012: 20-40.

Salazar, Boris. Qué hacer con la educación: algunas ideas para comenzar (I), Razón Pública, 12 de diciembre de 2011. http://www.razonpublica.com/index. php/econom\%C3\%ADa-y-sociedad/261l-ique-hacer-con-la-educacion-algunas-ideas-para-comenzar-segunda-parte.html (último acceso: 22 de septiembre 2015).

De Sousa, Boaventura. «La universidad del siglo XXI. Para una reforma democrática y emancipadora de la universidad». Umbrales, $n^{\circ}$ 15, 2007: 13-70.

Winston, Gordon. «The Economic Structure of Higher Education: Subsidies, Customer Inputs, and Hierarchy». Discussion paper (Williams College), $\mathrm{n}^{\circ} 40$, 1996: 1-39.

Winston, Gordon. Why can't a college be more like a firm?, 1997. http://sites.williams.edu/wpehe/files/2011/06/DP-42.pdf (último acceso: 15 de noviembre de 2014).

Winston, Gordon. "Grow the college? Why bigger may be far from better». Discussion paper (Williams College) $\mathrm{n}^{\circ}$ 60, 2001: 1-10.

Winston, Gordon, Jared Carbone y Ethan Lewis. "What's been happening to higher education?». Discussion paper (Williams College), n 17, 1998: 1-43.

World Commission on Environment and Development (WCED). Our common future. Nueva York: UN, 1987. 